\title{
High Space-Resolutive Evaluation of Subsurface Stress Distribution by Strain Scanning Method with Analyzer Using High-Energy Synchrotron X-Rays*
}

\author{
Takahisa SHOBU**, Jun'ichiro MIZUKI**, Kenji SUZUKI***, \\ Yoshiaki AKINIWA*** and Keisuke TANAKA***
}

\begin{abstract}
The surface aberration effect in the strain scanning method with a Ge analyzer was examined using high- energy X-rays from the undulator synchrotron source. The synchrotron $\mathrm{X}$-rays from the undulator source had an enough intensity for the strain scanning method using a goniometer with the analyzer. The use of a Ge (111) analyzer showed remarkable reduction of the surface aberration effect. However, there still existed the surface aberration for the very-near surface region from the surface to the depth of $50 \mu \mathrm{m}$. A correction method was proposed by taking into account of the effects of the divergence of the Ge analyzer, the mis-setting of the analyzer and the X-ray attenuation. The proposed correction method was very useful for eliminating the surface aberration effect. The correction method enables a high space-resolutive evaluation of the subsurface stress distribution. The method was successfully applied to the determination of the residual stress distribution of the shot-peened steel. A precise $d_{0}$ value of the strain-free lattice spacing necessary was determined from the surface stress measured by the conventional $\sin ^{2} \psi$ method using $\mathrm{Cr}-\mathrm{K} \alpha$ radiation.
\end{abstract}

Key Words: Residual Stress, Experimental Stress Analysis, Nondestructive Inspection, XRay Stress Measurement, Synchrotron Radiation, Strain Scanning Method

\section{Introduction}

The subsurface distribution of the residual stress has a large influence on crack initiation and propagation in stress corrosion cracking as well as in fatigue. To guarantee the reliability of structural components against stress corrosion cracking and fatigue, a non-destructive method is required to determine a precise distribution of the stress to the depth of few hundreds micrometers below the surface. The conventional method of X-ray stress measurement using $\mathrm{Cr}-\mathrm{K} \alpha$ radiation only detects the stress residing in the surface layer of a few tens micrometers, and the

* Received 25th November, 2005 (No. 05-4261)

** Synchrotron Radiation Research Unit, Quantum Beam Science Directorate, Japan Atomic Energy Agency, Kouto, Sayo-cho, Sayo-gun, Hyogo 679-5148, Japan.

E-mail: shobu@sp8sun.spring8.or.jp

*** Department of Technology and Living Sciences, Niigata University, 8050 Igarashi-2no-cho, Niigata 950-2181, Japan

**** Department of Mechanical Engineering, Nagoya University, Furoh-cho, Chikusa-ku, Nagoya 464-8603, Japan repetition of surface removal is necessary to determine the subsurface stress distribution. While neutron diffraction can detect the stress in the interior of samples, the spatial resolution is limited.

High-energy X-rays from a synchrotron source have a large penetration depth which reaches $1.2 \mathrm{~mm}$ for a steel in the case of X-rays with an energy of $70 \mathrm{keV}$. Furthermore, the synchrotron X-rays have very high intensity and small divergence, which enable a high spatial resolution in stress measurements. Several methods have been proposed to determine the subsurface stress distribution using high-energy X-rays. Akiniwa et al. proposed the constant penetration method and utilized the $\sin ^{2} \psi$ method for stress determination. The method is time-consuming, though it is accurate. Webster and others proposed a strain scanning method to determine the strain distribution in the sample $^{(1)}$. In the strain scanning method, the strain distribution is determined by scanning a small gauge volume which is determined by the sizes of the X-ray beam and the slits. This method has the following advantages:

1. The measurement time is shorter than that by a 
conventional $\sin ^{2} \psi$ method ${ }^{(2)}$.

2. The three dimensional stress can be measured from the measured strains.

However, to improve the accuracy of the strain scanning method, the following problems should be solved:

1. To clarify the optical systems for an accurate stress measurement.

2. To remove the error due to the surface aberration effect $^{(3)}$.

3. To determine the strain without knowing a precise strain-free lattice spacing.

The shift of the diffraction angle due to partial sinking of the diffracting gauge volume beneath the surface is called the surface aberration effect. Recently, Machiya and others studied the surface aberration effect in the strain scanning method using X-rays from the bending magnet source and proposed the correction method for the cases of the optical systems with double slits ${ }^{(4)}$. Although an accurate measurement of the subsurface strain distribution became possible by their correction method, spatial resolution is rather limited because of a fairly large divergence of the double slits. As another method, Withers and others proposed to use an analyzer to reduce the error due to the surface aberration effect. The amount of reduction of the error by the introduction of an analyzer is not yet clarified. The strain scanning method using an analyzer is only available in undulator or wiggler beam-lines and is not possible for bending magnet beam-lines, because of the severe attenuation of the X-ray intensity by the analyzer. The gauge volume determined by the analyzer is very small compared with that by the double slits optics, so that a higher space-resolution is expected.

In the present study, the reduction of the surface aberration effect by the analyzer is examined and a new correction method is proposed to remove the surface aberration effect.

\section{Correction Method}

\subsection{Analysis of stress and strain}

The axes of the stress, $\sigma$, and strains, $\varepsilon$, are defined as shown in Fig. 1, where the $\sigma_{3}$ (or $\varepsilon_{3}$ ) is perpendicular to the surface. The stress-strain relations for isotropic materials are given by

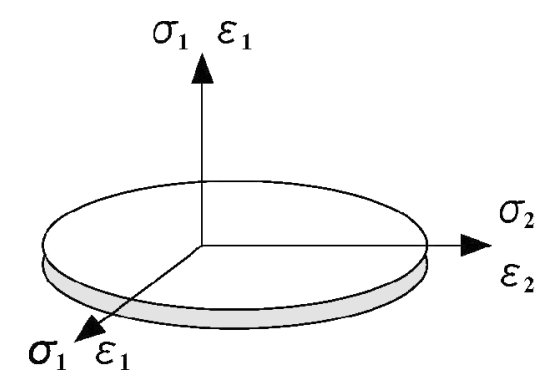

Fig. 1 Coordinate system for stress and strain

$$
\begin{aligned}
& \varepsilon_{1}=\frac{1}{E}\left[\sigma_{1}-v\left(\sigma_{2}+\sigma_{3}\right)\right] \\
& \varepsilon_{2}=\frac{1}{E}\left[\sigma_{2}-v\left(\sigma_{3}+\sigma_{1}\right)\right] \\
& \varepsilon_{3}=\frac{1}{E}\left[\sigma_{3}-v\left(\sigma_{1}+\sigma_{2}\right)\right]
\end{aligned}
$$

where $E$ is Young's modulus and $v$ is Poisson's ratio for a specimen. When the equi-biaxial stress state $\left(\sigma_{1}=\sigma_{2}\right)$ is assumed, the above equations are rewritten as follows:

$$
\begin{aligned}
\sigma_{1} & =\frac{E}{(1+v)(1-2 v)}\left(\varepsilon_{1}+v \varepsilon_{3}\right) \\
\sigma_{3} & =\frac{2 v E}{(1+v)(1-2 v)}\left(\varepsilon_{1}+\frac{1-v}{2 v} \varepsilon_{3}\right)
\end{aligned}
$$

In addition, when the plane stress is assumed, $\sigma_{3}$ is zero. As a result, the next equation is derived.

$$
\sigma_{1}=-\frac{E}{2 v} \varepsilon_{3}
$$

The relation between the lattice spacing, $d$, and the strain normal to the diffraction plane, $\varepsilon$, is given by

$$
\varepsilon=\frac{d-d_{0}}{d_{0}}
$$

where $d_{0}$ is the stress-free lattice spacing. The relation between the lattice spacing, $d$, and the diffraction angle, $2 \theta$, is given by Bragg's law as

$$
d=\frac{\lambda}{2 \sin \theta}
$$

where $\lambda$ is the wavelength.

\section{2 Correction of surface aberration effect}

In the strain scanning method, the strain distribution beneath the surface is obtained as follows:

1. As shown in Fig. 2, an instrumental gauge volume is determined by the incident and receiving slits, and the shape ideally is a rhombus.

2. X-ray is irradiated, and diffracted X-ray from the specimen contained in the gauge volume is measured.

3. The specimen is moved in the normal direction $z$ (The gauge volume is sunk in the specimen).

4. The measurement of the diffraction angle, $2 \theta$, is repeated, while scanning the position of the gauge volume in the depth direction $z$.

However, the actual gauge volume expands due to the divergence of the receiving slits. When the gauge volume is near the surface, the shift of the diffraction angle is caused by the difference in the centroid between the instrumental gauge volume and the actual gauge volume.



Fig. 2 Instrumental gauge volume by double slits 
The use of an analyzer is effective to reduce the surface aberration effect as reported by Withers and others ${ }^{(3)}$. The typical optical system of the strain scanning method with the analyzer is shown in Fig. 3. The divergence of the Ge (111) analyzer is very small compared with that of the double slits, so that the high space-resolution is expected.

Referring to Fig. 4, the causes of the surface aberration effect are assumed as follows:

1. The expansion of the gauge volume due to the divergence of the $\mathrm{Ge}$ analyzer.

2. The difference in the center line between the instrumental gauge volume and the analyzer due to the missetting of the analyzer.

3. The effect of the beam attenuation within the actual gauge volume.

In the strain scanning method by neutrons, the shift of the centroid due to the beam attenuation has been examined by Webster ${ }^{(5)}$, however, the expansion of the gauge volume and mis-setting of the analyzer were not taken into consideration. The centroid of the actual gauge volume shifts because of the change in diffraction intensity within the actual gauge volume, and moves from the geometric centroid, O, to the actual centroid, G, as shown in Fig. 5 . The values of shift $u$ and $v$ are obtained from the ratio of the intensity moment to the intensity for each direction as

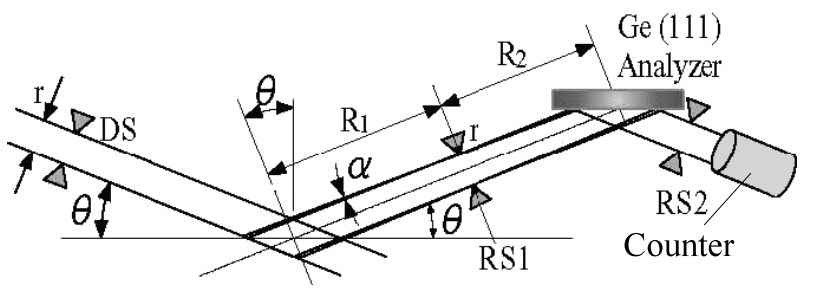

Fig. 3 Optics of strain scanning method with analyzer

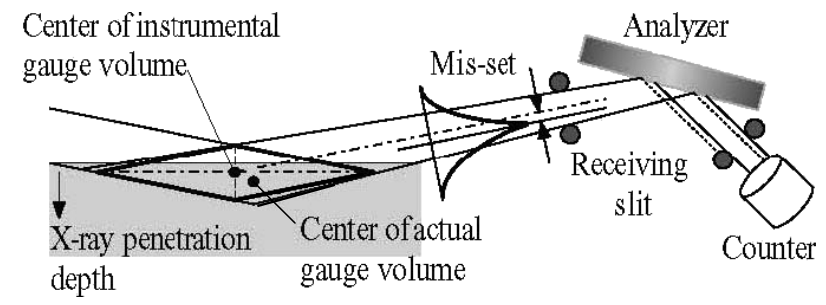

Fig. 4 Shift of centroid due to surface aberration effect

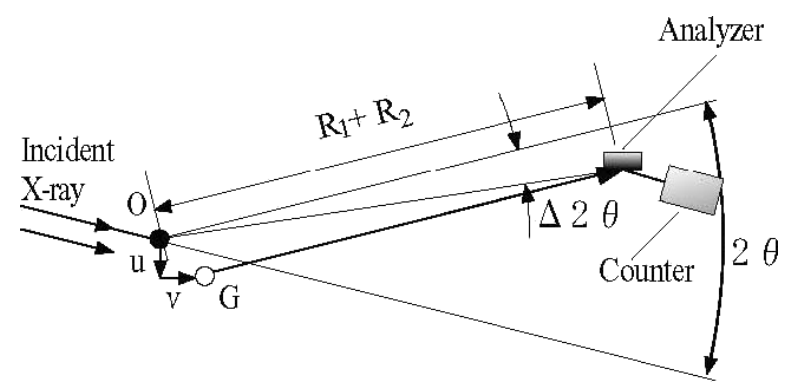

Fig. 5 Angle shift due to shift of centroid follows:

$$
u=\frac{\int_{V} I x d x}{\int_{V} I d x}, \quad v=\frac{\int_{V} I y d y}{\int_{V} I d y}
$$

where $I$ is the actual intensity at the position, $x$ and $y$ are the vertical and horizontal distance from the centroid of the instrumental gauge volume, $\mathrm{O}$. The integral is taken over the whole of the actual gauge volume, $V$.

When the actual centroid of the diffraction intensity shifts as shown in Fig. 5, this shift causes the angle shift of the measured diffraction angle which is denoted by $\Delta 2 \theta$.

\section{Experimental Procedure}

\subsection{Materials and specimens}

The experimental material was $\mathrm{S} 45 \mathrm{C}$ (steel with $0.45 \%$ carbon). The specimen was a flat plate with a thickness of $4 \mathrm{~mm}$. The reference specimen was annealed and stress-free. The other specimen was shot-peened to introduce a compressive residual stress in the surface layer. These annealed and shot-peened specimens were the same specimens used in our previous study ${ }^{(6)}$. The distributions of the residual stress were measured by the surfaceremoval method combined with the conventional $\sin ^{2} \psi$ method. The residual stress of the shot-peened specimen was in an equi-biaxial plane stress state.

\section{2 Conditions for synchrotron X-rays}

The beam line used for the experiment was the beam line BL22XU of JAEA (Japan Atomic Energy Agency) at SPring- $8^{(7)}$. The synchrotron source of the beam line comes from the undulator insertion source, and has very strong intensity and high-energy. The high brightness and high resolution of X-ray source can be obtained by a monochromator cooled with liquid nitrogen. The change of the brightness can be kept $0.3 \%$ or less by $\operatorname{MOSTAB}^{(8)}$. The intensity of the beam is $6 \times 10^{10}$ photons/sec. The synchrotron X-ray beam was focused by Be lenses and the size of the beam at the position of $120 \mathrm{~m}$ from synchrotron source was $0.6 \mathrm{~mm}$ in height and $1.2 \mathrm{~mm}$ in width.

A 4-circle goniometer of a horizontal type was used and is excellent in measurement accuracy, because horizontal goniometer is not affected by gravity. The specimen was set on the $z$-stage attached to the 4-circle goniometer. The X-ray conditions for the strain scanning method are summarized in Table 1. The X-ray energy was $69.503 \mathrm{keV}$ and the irradiated area made by the divergent slit was $1.0 \mathrm{~mm} \times 0.2 \mathrm{~mm}$. The Ge (111) was used as the analyzer crystal which has good crystallinity to ensure a high spatial resolution.

The diffraction of Fe 211 was measured and the X-ray elastic constants calculated by Kröner model ${ }^{(9)}$ was used for stress calculation. During measurement, the specimen was rotated in order to increase the number of the diffracting grains. 
Table 1 X-Ray conditions for strain scanning method

\begin{tabular}{l|c}
\hline Wavelength & $0.1783 \AA$ \\
Energy & $69.503 \mathrm{keV}$ \\
Monochromatic crystal & $\mathrm{Si}(111)$ \\
Sizes of divergent slits & Height $=1.0 \mathrm{~mm}$ \\
& Width $=0.2 \mathrm{~mm}$ \\
Sizes of receiving slits & Height $=1.0 \mathrm{~mm}$ \\
& Width $=0.2 \mathrm{~mm}$ \\
Length between DS and O (R0) & $6000 \mathrm{~mm}$ \\
Length between O and RS1 (R1) & $300 \mathrm{~mm}$ \\
Length between RS1 and Ge (R2) & $850 \mathrm{~mm}$ \\
Length between Ge and RS2 & $40 \mathrm{~mm}$ \\
Length between RS1 and RS2 & $890 \mathrm{~mm}$ \\
Analyzer & Ge (111) \\
\hline Specimen & Annealed S45C \\
& Shot-peened S45C \\
\hline Diffraction & Fe 211 \\
Diffraction angle $\theta$ & $8.7399 \mathrm{deg}$ \\
X-ray Young's modulus $E$ & $223 \mathrm{GPa}$ \\
X-ray Poisson's ratio $v$ & 0.273 \\
\hline
\end{tabular}

\section{Results and Discussion}

\subsection{Use of analyzer to reduce the surface aberra- tion effect}

Figure 6 shows the diffraction angle measured using the strain scanning method as a function of depth, $z$, for the annealed specimen. The diffraction angle, $2 \theta$, should be constant, because there is no strain in the annealed specimen. The diffraction angle measured using the double slits method without analyzer shifts greatly near the surface as seen in Fig. 6, because the divergence of the double slits is large. This angle shift near the surface is due to the surface aberration method. On the other hand, the shift of the diffraction angle measured with the analyzer is small and only occurs very near the surface. Although the angle shift in this case is very small compared with the case of the double slits method, there still exists a small angle shift from the surface to the depth of $50 \mu \mathrm{m}$. Therefore, the correction method for the surface aberration effect with the analyzer is considered in the next section and an accurate and convenient stress scanning method will be proposed by combining Ge analyzer usage with the correction of the surface aberration effect.

In the strain scanning method, the measurement of the $d_{0}$ value, which is the lattice spacing without strain, is always required to calculate a strain and is very significant to achieve high accuracy. In industrial applications of the strain scanning method, a direct determination of the $d_{0}$ value is often difficult. To circumvent this difficulty, the $d_{0}$ value can be determined from the surface stress measured by the conventional $\sin ^{2} \psi$ method of X-ray stress measurement by $\mathrm{Cr}-\mathrm{K} \alpha$ as follows. When the stress state is equi-biaxial plane stress, the $d_{0}$ value is determined by Eqs. (3) and (4), as

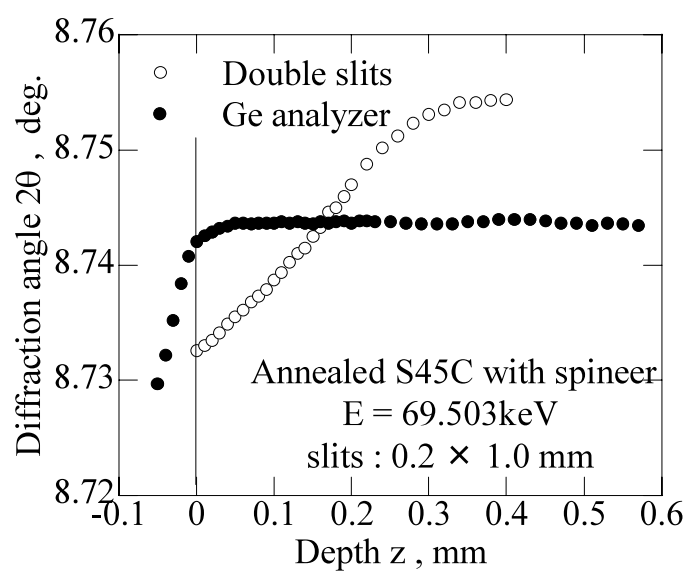

Fig. 6 Reduction of the surface aberration effect by analyzer

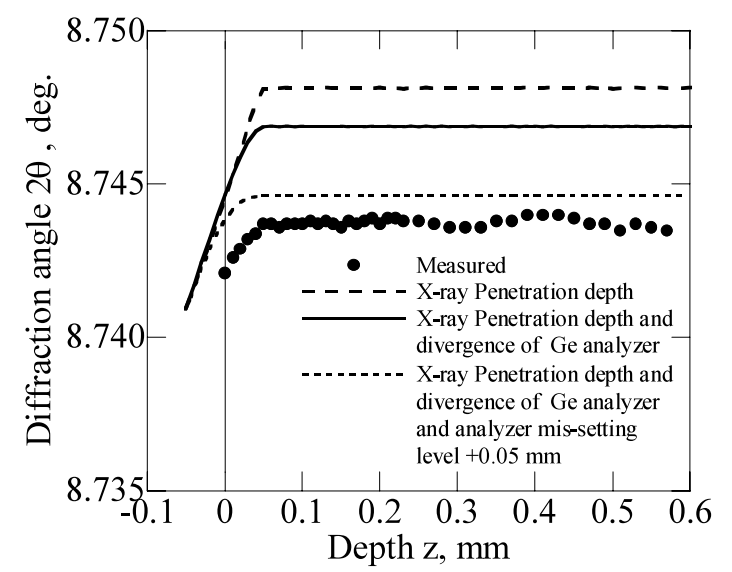

Fig. 7 Surface aberration effect for goniometer with analyzer

$$
d_{0}=-\frac{E}{E-2 v \sigma_{0}} d
$$

The $d_{0}$ value is determined by substituting the stress value, $\sigma_{0}$, determined by the conventional $\sin ^{2} \psi$ method of stress measurement and the measured lattice spacing on the surface, $d$.

\subsection{Correction of surface aberration of strain scanning method with analyzer}

As the above mentioned, the following three causes are responsible for the surface aberration effect: the X-ray penetration depth, the extension of the gauge volume and the mis-setting of the Ge analyzer. Figure 7 shows the calculated and measured values of the diffraction angle as a function of depth.

The broken line shows the result calculated by taking into account of the surface aberration effect by X-ray attenuation in the instrumental gauge volume. The calculated diffraction angle decreases more rapidly near the surface compared with the measured one. Next, the effect of divergence of the $\mathrm{Ge}$ analyzer was considered in addition to the X-ray attenuation effect. The divergent angle of the Ge analyzer was defined as the full width at half maximum (FWHM) of the rocking curve of the analyzer. The calculated line shown with the solid line gets closer to 


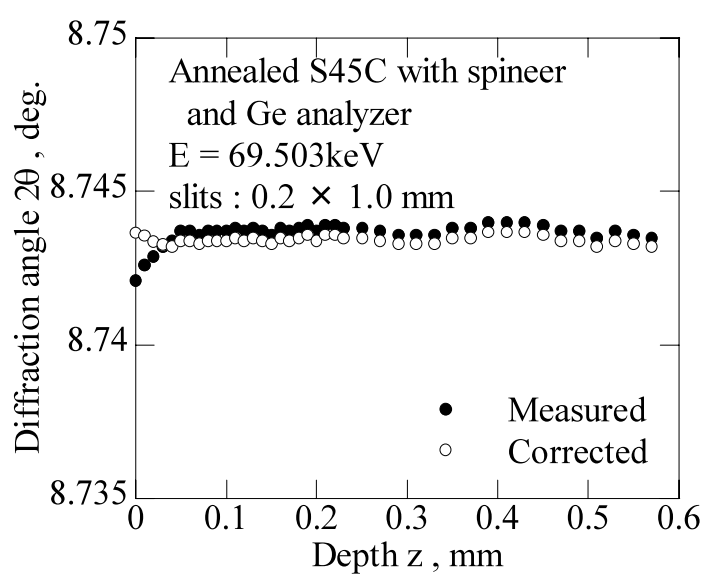

Fig. 8 Corrected and measured diffraction angles

the measured results. The relation obtained after all three corrections is shown with the dotted line in Fig. 7. The agreement with the measured relation is much improved.

From the above comparisons, it can be concluded that three causes, the X-ray attenuation effect, the extension of the gauge volume due to the Ge analyzer and the missetting of the Ge analyzer, are all responsible for the surface aberration of the measured diffraction angles. Therefore the values of shift of diffraction angle, $\Delta 2 \theta$, obtained by calculation are deducted from measured values. The variation of the corrected diffraction angle, $2 \theta$, is shown in Fig. 8. The difference between the measured value and corrected value at surface is 0.002 degree and this value is equivalent to $60 \mathrm{MPa}$. The corrected angle is almost constant, which is appropriate for measurement of the annealed specimen.

\subsection{Application of strain scanning method with analyzer}

To examine our correction method proposed above, the distribution of the residual stress for the shot- peened specimen was measured. The measured diffraction angle was corrected by our method, and the result is shown in Fig. 9. The $d_{0}$ value is determined by Eq. (7). In Fig. 9, the open circles indicate the residual stress measured by the strain scanning method. The solid circles indicate the residual stress obtained in our previous study ${ }^{(6)}$, where the surface layer of the shot-peened specimen was removed successively by electropolishing and the stress measurement by the $\sin ^{2} \psi$ method using $\mathrm{Cr}-\mathrm{K} \alpha$ radiation was repeated. The value of the stress measured by $\sin ^{2} \psi$ method were corrected by taking account of the surface removal. With respect to the result by the strain scanning method with analyzer, the residual stress is a compression at the surface and shows the minimum beneath the surface, and approaches to zero in the deeper region. The surface layer of the compressive residual stress is about $0.3 \mathrm{~mm}$ in thickness. The distribution determined by the strain scanning method well agrees to that by the surface-removal method,

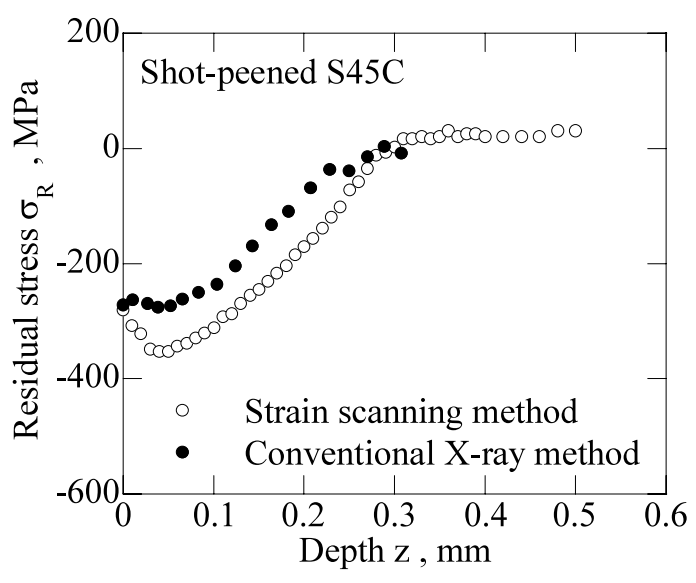

Fig. 9 Distribution of residual stress measured by strain scanning method and conventional X-ray method

though the value of the compressive residual stress for the strain scanning method is a little larger than that obtained by the surface-removal method.

\section{Conclusions}

The surface aberration effect in the strain scanning method with a Ge analyzer was examined using high- energy X-rays from the undulator synchrotron source. A new correction method was proposed to remove the surface aberration effect. The obtained results are summarized as follows;

1. The synchrotron X-rays from the undulator source had an enough intensity for the strain scanning method with the analyzer.

2. The use of the Ge (111) analyzer showed remarkable reduction of the surface aberration effect. However, there still existed the surface aberration for the very-near surface region from the surface to the depth of $50 \mu \mathrm{m}$.

3. A correction method was proposed by taking into account of the effects of the divergence of the Ge analyzer, the mis-setting of the analyzer and the X-ray attenuation. This correction method was very useful for removing the surface aberration effect.

4. The proposed correction method enables a high space-resolutive evaluation of the subsurface stress distribution. The method was successfully applied to the determination of the residual stress distribution of the shotpeened steel.

5. The $d_{0}$ value of the strain-free lattice spacing necessary for strain calculation was determined from the surface stress measured by the conventional $\sin ^{2} \psi$ method using $\mathrm{Cr}-\mathrm{K} \alpha$ radiation.

\section{Acknowledgements}

The authors are obliged to Dr. T. Inami and Dr. K. Ohwada (JAEA) for assistance of the experiments and helpful suggestions. 


\section{References}

( 1 ) Webster, P.J., Vaughar, G.B.M., Mills, G. and Kang, W.P., High Resolution Synchrotron Strain Scanning at BM16 at the ESRF, Mater. Sci. Forum, Vol.278-281 (1998), pp.323-328.

(2) Standard Method for X-Ray Stress Measurement, JSMS-SD-10-05, Soc. of Mater Sci., Japan, (2005), pp.3-11.

( 3 ) Withers, P.J., Preuss, M., Webster, P.J., Hughes, D.J. and Korsunsky, A.M., Residual Stress Measurement by Synchrotron Radiation, Mater. Sci. Forum, Vol.404407 (2002), pp.1-12.

(4) Machiya, S., Akiniwa, Y., Suzuki, K., Tanaka, K., Kurimura, T. and Koguma, H., Measurement of Residual Stress Distribution by Strain Scanning Method Using High Energy X-Rays from Synchrotron Source, J. of Soc. of Mater. Sci., Japan, (in Japanese), to be accepted.
( 5 ) Webster, G.A., Ed., Polycrystalline Materials - Determinations of Residual Stresses by Neutron Diffraction, ISO/TTA3 Technology Trends Assessment, Geneva, (2001).

(6 ) Akiniwa, Y., Tanaka, K., Suzuki, K., Yanase, E., Nishio, K., Kusumi, Y., Okado, H. and Arai, K., Evaluation of Residual Stress Distribution in Shot-Peened Steel by Synchrotron Radiation, J. of Soc. of Mater. Sci., Japan, Vol.52 (2003), pp.764-769.

( 7 ) http://www.spring8.or.jp/e/bl/BL22XU/index.html

( 8 ) Kudo, T., Nishino, Y., Suzuki, M., Tanida, H., Hirono, T. and Ishikawa, T., Stabilization of Synchrotron Radiation X-Ray Beam by MOSTAB, J. of Japanese Soc. for Synchrotron Radiation Research, (in Japanese), Vol.16 (2003), pp.173-177.

( 9 ) Kröner, E., Berechnung der Elastischen Konstanten des Vielkristalls aus den Konstanten des Einkristalls, Z. Physik., Vol.151 (1958), pp.504-518. 